\title{
Un análisis de las representaciones mediáticas y las desigualdades estructurales en el fútbol de mujeres en Argentina
}

\author{
An Analysis of Media Representations and Structural Inequalities in \\ Women's Football in Argentina
}

\author{
Verónica Moreira \\ Universidade de Buenos Aires (UBA), Buenos Aires/Argentina \\ Doutora em Ciências Sociais, UBA \\ veromoreira175@gmail.com \\ Martín Álvarez Litke \\ Universidade de Buenos Aires (UBA), Buenos Aires/Argentina \\ Doutorando em Antropologia, Faculdade de Filosofia e Letras, UBA
}

\begin{abstract}
Resumen: Este artículo analiza algunos aspectos de la posición subalterna de las atletas argentinas. El caso de las jugadoras que participan en la liga competitiva de la AFA (Asociación de Fútbol Argentino) expone las dificultades que estas deportistas enfrentan a diario cuando quieren desarrollar su práctica. El artículo estudia la relación conflictiva entre las deportistas, las instituciones deportivas y los medios de comunicación; una relación que pone de manifiesto la desigualdad y el trato discriminatorio hacia las futbolistas en dichos ámbitos. Para esto, por un lado, realizamos un diagnóstico de las representaciones de las futbolistas en el diario Olé, medio de comunicación deportivo online que aún publica ejemplares en versión papel; y, por otro lado, analizamos los datos de la observación realizada durante el trabajo de campo en el Club Atlético Boca Juniors, así como también las entrevistas a sus jugadoras y a representantes de otros equipos de la primera división.
\end{abstract}

Palabras Clave: Fútbol; Mujeres; Desigualdad; Condiciones estructurales; Medios de comunicación.

ABSTRACT: This article analizes various aspects of the subordinate position of Agentina's women athletes. The case of the footballers who participate in the Argentine Football Association's competitive league exposes the difficulties these sportswomen face every day when they wish to practice their sports. The article studies the conflictive relationship between sportswomen, sporting institutions and the media; a relationship that highlights the inequality and discrimination towards women footballers. Thus, on the one hand, we review the representations of women footballers in Olé, a daily newspaper dedicated to sports, with an online version along with a paper version; and on the other hand, we analize data obtained from observations made as part of the field work in Club Atlético Boca Juniors, along with interviews with players from this and other first division football clubs.

KEYWORDS: Football; Women; Media; Structural Conditions; Inequality. 
FuLiA / UFMG - [...] las desigualdades estructurales en el fútbol de mujeres en Argentina

\section{INTRODUCCIÓN}

Las noticias sobre el fútbol practicado por mujeres ${ }^{1}$ en Argentina se multiplicaron en los medios de comunicación masivos, especialmente, a partir de la participación que el seleccionado nacional tuvo en la Copa América disputada en Chile en abril de 2018. Las jugadoras obtuvieron un valioso e inesperado tercer puesto en un torneo que enfrentó a diez países. En el tramo final del campeonato, antes de disputar el partido contra la selección de Colombia, las atletas posaron de una manera singular: cada una tomó su oreja derecha en señal de protesta para denunciar la discriminación que sufrían de parte de la AFA (Asociación del Fútbol Argentino) por el hecho de ser mujeres. El equipo argentino ganó el partido frente al conjunto colombiano que era más poderoso y, al día siguiente, la foto de las jugadoras tomándose la oreja circuló rápidamente por las redes sociales y los medios de comunicación.

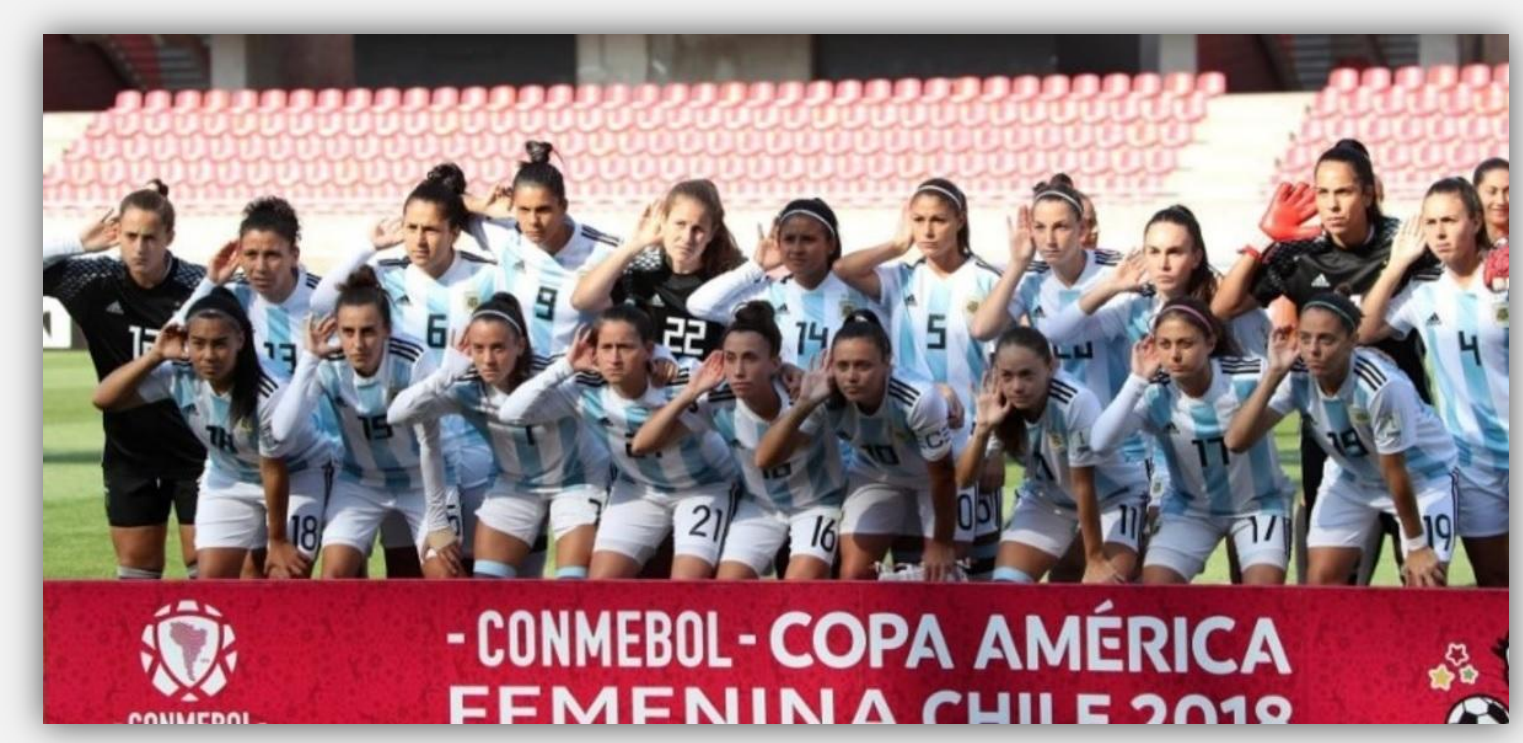

Site: https://www.elcohetealaluna.com/el-topo-gigio-tiene-cara-de-mujer.

\footnotetext{
${ }^{1}$ GOELLNER; SILVA; BOTELHO-GOMES. A sub-representação do futebol praticado por mulheres no jornalismo esportivo de Portugal: um estudo sobre a Algarve Women's Football Cup. KESSLER, Futebol ou futebóis: é plural ou singular?. Coincidimos con las autoras en el uso del término "fútbol practicado por mujeres" para indicar adrede que el fútbol es una misma práctica que puede ser adoptada por personas con distintas identidades de género. De todos modos, en el artículo también usamos de manera indistinta el término "fútbol femenino".
} 
FuLiA / UFMG - [...] las desigualdades estructurales en el fútbol de mujeres en Argentina

Los reclamos de la selección de fútbol cobraron visibilidad raudamente, vitalizados y amplificados por las demandas que las mujeres realizaban en otros ámbitos de la vida pública. En este sentido, cabe señalar que 2018 fue un año de particular importancia para los movimientos de mujeres en la Argentina ya que el debate legislativo en torno al Proyecto de Ley de Interrupción Voluntaria del Embarazo catalizó la discusión respecto a los derechos de las mujeres en todos los sectores sociales.

El deporte no ha sido ajeno a estas discusiones. El caso de las jugadoras de fútbol, como el de tantas otras atletas, plantea una situación conflictiva en lo que refiere a la relación entre las deportistas, las instituciones deportivas y los medios de comunicación; una relación que pone de manifiesto la desigualdad y el trato discriminatorio que reciben las mujeres por parte de sus clubes y de los medios cuando (no) elaboran noticias sobre sus actuaciones.

Este artículo pretende analizar ciertas dimensiones de esta relación para dar cuenta de la posición subalterna de las atletas en el mundo deportivo. Por un lado, realizamos un primer diagnóstico de las representaciones de las futbolistas en un medio de comunicación reconocido en Argentina, el diario Olé. Estudiamos su versión en papel así como la versión online durante abril de 2018, mes en el que se disputó la Copa América de Fútbol Femenino. Además, completamos la información con los ejemplares del mes del Mundial de Fútbol de varones organizado en Rusia en el mismo año. Por otro lado, analizamos los datos construidos a partir del trabajo de campo llevado a cabo en el Club Atlético Boca Juniors entre 2016 y 2018, así como las entrevistas a sus jugadoras y de otros equipos de la primera división de los torneos organizados por la AFA.

En función de nuestro objetivo el trabajo se organiza en tres partes: una primera parte destinada a presentar las líneas de investigación desarrolladas en las Ciencias Sociales y Humanas sobre el deporte para identificar cuál ha sido el lugar de los estudios de género en dichas discusiones; una segunda sección que analiza las representaciones mediáticas de las deportistas en el único diario deportivo de tirada 
FuLiA / UFMG - [...] las desigualdades estructurales en el fútbol de mujeres en Argentina

nacional; y una tercera parte en la que exponemos las condiciones de la práctica deportiva desde el punto de vista de las futbolistas. ${ }^{2}$

\section{ESTUDIOS SOCIALES DEL DEPORTE}

El año 1984 es clave para los estudios sociales del deporte en Argentina, ya que en esa fecha se publicó el primer texto que tomó al fútbol como objeto de análisis. A partir de los cantos de los hinchas, Eduardo Archetti observó cómo éstos construían un tipo de masculinidad que se apoyaba fuertemente en la valentía, el coraje, la dominación y la subordinación del rival. ${ }^{3}$ Además, Archetti investigó la relación entre el deporte y la identidad nacional considerando no sólo el fútbol sino también otras disciplinas como el polo, el automovilismo y el boxeo. ${ }^{4}$ La pregunta central que articuló estos estudios fue la de cómo dichos deportes permitían la elaboración de relatos sobre la patria.

Desde la década del noventa del siglo pasado, con el reconocido sociólogo Pablo Alabarces se afianzaron una serie de investigaciones sobre el deporte, que problematizaron aspectos de estos dos grandes ejes de discusión: las prácticas violentas de los hinchas en pos de su masculinidad y las narraciones legítimas de la nación. ${ }^{5}$ Ahora bien, con excepción de pocas autoras que reflexionaron a fines de los años noventa y principio de la década de 2000 sobre el comportamiento de las espectadoras en los estadios de fútbol,6 las mujeres han sido sistemáticamente relegadas de los análisis académicos. Los estudios referentes del campo del deporte se han producido principalmente sobre y desde la perspectiva de los varones en sus distintos roles: como deportistas, hinchas y dirigentes.

\footnotetext{
${ }^{2}$ El trabajo es producto de las exposiciones de los autores en el Primer y Segundo Encuentro de la Red de Investigación sobre Fútbol y Mujeres realizados el 24 y 25 de septiembre en San Pablo y el 23 y 24 de noviembre de 2018 en Buenos Aires, con el apoyo de Arts and Humanities Research Council, Reino Unido.

${ }^{3}$ ARCHETTI. Fútbol y ethos.

${ }_{5}^{4}$ ARCHETTI. El potrero, la pista y el ring - las patrias del deporte argentino.

${ }^{5}$ ALABARCES. Fútbol y patria.

${ }^{6}$ BINELLO Y DOMINO. Mujeres en el área chica; Binello, Conde, Martínez y Rodríguez. Mujeres y fútbol: ¿territorio conquistado o a conquistar; Conde y Rodríguez. Mujeres en el fútbol argentino: sobre prácticas y representaciones; Conde. El poder de la razón: las mujeres en el fútbol.
} 
FuLiA / UFMG - [...] las desigualdades estructurales en el fútbol de mujeres en Argentina

La ausencia de los estudios sobre las deportistas comenzó a revertirse en los últimos años gracias a las investigaciones de un conjunto de jóvenes cuyo interés es el abordaje del fútbol competitivo. ${ }^{7}$ Estudiar mujeres desde la perspectiva de género implica no sólo darles la palabra y visibilizar sus actuaciones como protagonistas de los escenarios deportivos, discutir las arbitrariedades y discriminaciones que han sufrido y sufren a diario, sino también observar la manera en que las jugadoras negocian con estas dificultades y construyen sus identidades como atletas. En este sentido, algunas preguntas que guían estas investigaciones giran en torno a los siguientes tópicos: ¿Cuáles son las condiciones estructurales de la práctica del fútbol femenino? ¿Qué imaginarios sociales existen sobre las mujeres que juegan al fútbol? ¿Cómo son representadas? ¿Cuáles son las representaciones de las propias jugadoras sobre su práctica y cómo negocian con los discursos sobre ellas? ¿Qué lugar juega el deporte en su construcción de identidad? ¿Cómo se construye la relación entre fútbol femenino e identidad nacional en un país futbolcéntrico como Argentina? ¿De qué manera impactan las discusiones del feminismo en el fútbol jugado por mujeres?

Estas investigaciones comienzan a saldar aspectos críticos de los estudios sobre deporte y nación, pues no sólo problematizan la participación de las mujeres en el fútbol en la actualidad, sino que indagan respecto del lugar que las mismas han ocupado en la historia de esta disciplina. La escasez de relatos en documentos oficiales y fuentes periodísticas respecto de la actuación de las deportistas desde la fundación del fútbol acarrea dos consecuencias. La primera es de orden metodológico, en tanto que la falta de registros de la práctica obstaculiza -aunque no impide- una reconstrucción más democrática de la historia del deporte y el fútbol en nuestro país. Cabe señalar, que esta dificultad se reforzó por el enfoque adoptado por los estudios sociales del deporte que excluyeron a las mujeres de las preguntas de investigación. La segunda consecuencia, de orden cultural y derivada de la primera, es que la

\footnotetext{
${ }^{7}$ GARTON. Las Guerreras: Futbolistas, estudiantes, trabajadoras. Un estudio sobre el fútbol de mujeres en el Club Deportivo UAl Urquiza; GARTÓN E HIJÓS. La deportista moderna: género, clase y consumo en el fútbol, running y hockey argentinos; IBARRA. Lógicas del fútbol femenino: sin salario ni clubes; ALVAREZ LITKE. Marcando la cancha: una aproximación al fútbol femenino desde las ciencias sociales.
} 
FuLiA / UFMG - [...] las desigualdades estructurales en el fútbol de mujeres en Argentina

negación de las mujeres jugadoras de fútbol ha creado y sostenido la falacia de que el fútbol es un deporte únicamente para varones. ${ }^{8}$

\section{MEDIOS Y DEPORTISTAS EN CONFLICTO}

Como explicamos, la academia ha ignorado a las deportistas hasta épocas muy recientes. Por su parte, los medios de comunicación no les han dispensado un trato muy diferente. En este sentido, la encuesta realizada por Magalí Robles expone sin dejar dudas cuál ha sido históricamente la posición que las atletas han ocupado en los medios de comunicación dedicados al deporte. Robles analizó las tapas de la revista El Gráfico $^{9}$ desde su fundación en 1919 hasta su cierre definitivo en enero de 2018. A modo de ilustración rescatamos algunos datos representativos de dicha estadística. Durante los primeros cincuenta años, sólo el 6\% de las tapas fue dedicado a las atletas. Desde 1964 hasta 1982 no hubo una sola mujer en la portada. Luego de estos 18 años, la reaparición de la presencia femenina se dio en enero de 1983, pero la tapa no estuvo dedicada exclusivamente a ésta. La portada reunión a tres mujeres rodeadas de 17 varones, todos campeones del año anterior. Otro aspecto relevante que aporta Robles es que las mujeres de las tapas tuvieron que ser campeonas para estar allí; criterio que no fue usado para justificar la presencia de los atletas. ${ }^{10}$ Es decir que podemos hablar de la excepcionalidad de los casos en los que las mujeres deportistas han sido visibilizadas en los medios de comunicación.

A continuación, analizamos de qué manera son presentadas las deportistas en la actualidad, centrándonos en el principal medio dedicado al deporte. Olé es el único diario deportivo en formato impreso de circulación nacional, que tiene gran influencia

8 Esta tendencia comienza a modificarse a partir del reconocimiento de los medios de comunicación a Las Pioneras del Fútbol Femenino en Argentina, un grupo de ex jugadoras que decidieron recopilar la historia del fútbol de mujeres y reconocer a las jugadoras de las décadas anteriores (desde 1950 hasta 1990). En especial, las Pioneras recuperaron la historia del equipo que viajó a México en 1971 para representar a la Argentina en el primer mundial de fútbol femenino (no oficializado por la FIFA).

9 Revista prestigiosa dedicada a los deportes, en sus primeros años estuvo dedicada al interés general.

${ }^{10}$ ROBLES. Volver a la Historia. 
FuLiA / UFMG - [...] las desigualdades estructurales en el fútbol de mujeres en Argentina

en Buenos Aires y ciudades cercanas, y por ello establece la agenda de noticias sobre deporte en otros medios de comunicación. Es el quinto diario en la lista de los más vendidos (21.262 ejemplares por día) ${ }^{11}$ y el quinto diario online más visitado (7.254.000 visitas). ${ }^{12}$

Respecto a las representaciones de las deportistas en el diario encontramos resultados similares a un trabajo anterior y a los de Robles: la subrepresentación. ${ }^{13}$ Es notable la ausencia de noticias sobre el campeonato oficial de la AFA, que se disputa desde 1991 y congrega a equipos de la Ciudad de Buenos Aires y el Conurbano Bonaerense en dos categorías, A y B. Si bien la estructura del diario responde a una cobertura centralmente futbolística en sus distintas variantes (fútbol de la primera división, de ascenso, internacional), el campeonato de las futbolistas no ha sido objeto de análisis a lo largo de estos años.

En el mismo sentido, encontramos una escasez de información en Olé sobre el desempeño de la Selección de Fútbol Femenino en la Copa América disputada en Chile. La Copa América estuvo organizada en dos etapas. La etapa inicial dividida en dos grupos de cinco equipos que se enfrentaron entre sí. Los dos primeros de cada grupo clasificaron para competir en un cuadrangular final. La Selección argentina jugó los días 05, 07, 09 y 13 de abril. Ganó todos los partidos a excepción del primero que perdió contra Brasil. El resto de los partidos jugó contra Bolivia, Ecuador y Venezuela. Las jugadoras argentinas pasaron a la ronda final y jugaron los días 16, 19 y 22 de abril en el cuadrangular contra Colombia, Brasil y Chile, respectivamente. Pese a la gran cantidad de partidos disputados por el seleccionado, el diario Olé publicó la primera noticia en su versión en papel el 18 de abril, luego del triunfo contra las atletas colombianas. En dicha nota, el diario - fiel a su estilo sensacionalista- no hizo referencia a los pormenores del juego sino al conflicto que las jugadoras habían entablado con los dirigentes de la AFA debido a las pésimas condiciones en las que

\footnotetext{
${ }^{11}$ Para el mes de mayo de 2018, según el Instituto Verificador de Circulaciones.

${ }_{12}^{12}$ Para el mes de junio de 2018, según ComScore MMX-Multi-plataform.

${ }^{13}$ ARAOZ ORTíZ; MOREIRA. Prensa deportiva en Argentina. Construcciones identitarias y estilos discursivos del deporte en el diario Olé.
} 
FuLiA / UFMG - [...] las desigualdades estructurales en el fútbol de mujeres en Argentina

desarrollaban sus entrenamientos. La nota también hacía referencia a un tweet de la arquera de la selección, donde criticaba a la AFA por contratar modelos publicitarias para presentar la camiseta oficial del equipo.

Las siguientes dos notas de Olé sobre la Copa América informaron brevemente que las deportistas perdieron contra las selecciones de Brasil y Chile. Sólo la última noticia relata algunos pasajes del enfrentamiento deportivo. Es decir, en torno a los siete partidos disputados, sólo uno de ellos recibió una cobertura en la que se mencionan hechos relativos a lo sucedido en el campo de juego.

Frente a esta (sub)representación de las mujeres futbolistas en el diario Olé, nos parece pertinente analizar cómo fueron presentadas las mujeres durante el Mundial de fútbol de varones de Rusia 2018. Durante esta competencia, aparecieron distintas notas donde se mostraron escenas de los jugadores en su tiempo libre junto a sus familias. En el marco de la sobresaturación de la información de la selección nacional de fútbol de varones, y de las selecciones más potentes del mundo, las mujeres aparecieron ocupando los roles cultural e históricamente asignados, vinculados al ámbito doméstico, la familia y la maternidad. En sintonía con las conclusiones sostenidas en otras investigaciones, ${ }^{14}$ las mujeres aparecen en el diario ocupando roles tradicionales, esto es, en calidad de esposas, madres y novias de los futbolistas.

Por otra parte, las mujeres en Olé también son representadas bajo los parámetros del otro estereotipo pensado y construido para ellas: la imagen de mujeres sexualizadas que aparecen en poses sensuales con bikinis o ropas que permiten el lucimiento de sus curvas para la mirada masculina. Las mujeres elegidas para la sección, que se llama "La Diosa del día”, responden a la feminidad hegemónica que estima positivamente cuerpos jóvenes, bellos, delgados y sensuales. ${ }^{15}$

La presencia de las mujeres en sus roles tradicionales (madres, esposas, novias y "diosas") y la subrepresentación de las deportistas (así como de las periodistas, conductoras, árbitras, y entrenadoras) frente a la saturación de información de sus

\footnotetext{
${ }^{14}$ BINELLO; DOMINO. Mujeres en el área chica.

${ }^{15}$ ARAOZ ORTÍZ; MOREIRA. Prensa deportiva en Argentina - construcciones identitarias y estilos discursivos del deporte en el diario Olé, p.121.
} 
FuLiA / UFMG - [...] las desigualdades estructurales en el fútbol de mujeres en Argentina

pares varones exponen la desjerarquización y subordinación de las mujeres en el diario. En relación con esto, un dato relevante asociado a la participación de las atletas en diferentes disciplinas es que, generalmente, Olé las describe, primero, como mujeres en sus funciones tradicionales y, luego, como deportistas. Es el caso de la tenista estadounidense multicampeona, Serena Williams, quién al pasar a octavos de final en Wimbledon, el diario tituló “Duelo de mamis”, subrayando su maternidad y la de su contrincante, Evgeniya Rodina. La nota fue acompañada por la foto de Serena con su hija en brazos. ${ }^{16}$

Al respecto, Jennifer Hargreaves sintetiza:

En términos generales, los medios de comunicación de masas informan sólo del deporte masculino presentándolo como una actividad "natural" asociada a la identidad masculina y al chauvinismo. La atención prestada al deporte femenino es, comparativamente hablando, mínima, y las ideologías deportivas convencionales en torno a las mujeres son consecuentes con las imágenes de la feminidad y de la maternidad. La prensa popular presenta casi siempre a las deportistas, primero, como mujeres y, después, como atletas. En esencia, éstas tienden a ser descritas a través de su sexualidad la belleza de sus piernas, su bonita figura- más que por sus capacidades técnicas o sus habilidades deportivas. ${ }^{17}$

La (in)visibilidad de la información da cuenta de la disputa por la imposición y la transmisión de los sentidos hegemónicos que circulan en nuestra sociedad. Por este motivo, la forma en que aparecen las mujeres en los medios deportivos no es inocente. Al presentarlas ocupando roles tradicionales, los medios refuerzan los estereotipos sobre las mujeres en el deporte y otros ámbitos obturando la posibilidad del público de acceder a otros modelos posibles de ser mujer y deportista.

Sin embargo, a pesar de esta presentación tendenciosa y de la escasez de información sobre el desarrollo de los encuentros deportivos que recabamos en nuestra investigación, la nota de Olé que presentó la foto de las jugadoras de la Selección protestando contra la AFA sirvió para visibilizar las desigualdades que sufrían -y aún padecen- las jugadoras argentinas. Más allá de que los medios de

\footnotetext{
${ }_{17}^{16}$ Diario Olé. Duelo de mamis, 07 jul. 2018.

${ }^{17}$ HARGREAVES. Promesa y problemas en el ocio y los deportes femeninos, p. 126.
} 
FuLiA / UFMG - [...] las desigualdades estructurales en el fútbol de mujeres en Argentina

comunicación hayan elegido publicar el hecho a partir de criterios de noticiabilidad sensacionalistas, las jugadoras encontraron allí la posibilidad de introducirse en los resquicios del discurso hegemónico y publicitar sus demandas en pos generar un cambio en su situación. Hoy, a casi un año de aquella acción, atestiguamos una proliferación de noticias sobre el fútbol practicado por mujeres, y la AFA anunció la profesionalización de la disciplina. Si bien hubo varios factores que influyeron en esto, la foto fue un puntapié inicial. Para comprender qué implicancias podría tener esta medida, en el siguiente apartado describimos las condiciones en las que las mujeres practican fútbol en Argentina.

\section{DEPORTISTAS EN EL CAMPO}

Como señalamos, la AFA anunció recientemente la profesionalización del fútbol femenino, que hasta el momento se mantiene como una disciplina amateur. Esto significa que las jugadoras no reciben un salario por llevar adelante su actividad en los clubes. Por este motivo, la mayoría de ellas, además de ser deportistas, trabajan y/o estudian carreras terciarias o universitarias, ya que reconocen que el fútbol no es una carrera que pueda sostenerlas económicamente en el presente ni mucho menos asegurar su situación material a futuro. Las implicancias de esto son que las jugadoras de fútbol en Argentina tienen menos tiempo para dedicarle a los entrenamientos y al descanso, en comparación con los jugadores varones y con las mujeres futbolistas de otros países. Esta situación genera un círculo del que es difícil escapar, pues el rendimiento y estado físico de las deportistas no responde según lo esperado para el nivel competitivo, y esto repercute en el nivel de juego y en las críticas que reciben las jugadoras por sus resultados deportivos. Así, se refuerza el prejuicio respecto de la calidad de juego inferior de las mujeres, lo que luego se utiliza para justificar una menor inversión en la disciplina. 
FuLiA / UFMG - [...] las desigualdades estructurales en el fútbol de mujeres en Argentina

Ahora bien, es necesario aclarar que las condiciones en las que las futbolistas desarrollan su práctica varían de un club a otro, dependiendo de la situación de cada institución y del lugar que ocupa el fútbol femenino en su estructura. Así, por ejemplo, mientras que en algunos clubes las jugadoras deben abonar una cuota social para poder participar de la actividad, en otros casos la entidad les otorga un "viático", es decir un monto de dinero para que puedan costear los traslados al predio de entrenamiento y a los partidos. Inclusive en el club UAI Urquiza, el más exitoso de los últimos años -ganó tres de los últimos cinco torneos en disputa-, las jugadoras reciben una beca para estudiar una carrera, y el club les consigue trabajo para que puedan mantenerse económicamente, aunque esto no se extiende a todas las integrantes del plantel. No podemos generalizar sobre todos los clubes, por lo cual introducimos algunas experiencias especialmente en torno al club Boca Juniors, complementando con datos de otras instituciones.

Boca Juniors es uno de los dos clubes más populares del país, y uno de los más poderosos económicamente. Su equipo de fútbol femenino ha participado del certamen de AFA desde su creación en 1991 y ha sido el más ganador de la competencia históricamente con un total de 23 títulos obtenidos en la máxima categoría.

Respecto de las condiciones que propone Boca Juniors para el fútbol femenino, su capitana expresaba en una entrevista:

yo creo que Boca es dentro de todo uno de los mejores. En eso no nos podemos quejar, porque nos dan obra social, no nos tenemos que preocupar por la ambulancia, por el policía, por si está el médico. Viste que hay otros equipos que tienen que vender rifas... eso corre todo por cuenta de Boca.

Tal como indica esta jugadora, Boca paga la obra social de las 30 jugadoras que integran su plantel, y se ocupa de los gastos fijos por partido que incluyen la seguridad en el estadio y los servicios de salud. Este no es un dato menor, ya que como nos explicaba Florencia, otra jugadora del plantel, en muchos casos los partidos se han suspendido porque un club no le pagó a la policía o a los médicos. La cobertura de la obra social es especialmente valorada por las jugadoras, ya que constantemente están expuestas al riesgo de lesionarse. En este sentido, Julieta nos relató cómo -cuando 
FuLiA / UFMG - [...] las desigualdades estructurales en el fútbol de mujeres en Argentina

entrenaba en otro club- tuvo que costearse su propia operación porque la institución no le pagaba la obra social (aunque esto había sido el trato convenido con antelación).

Además de la obra social, todas las jugadoras de Boca Juniors reciben un viático, aunque el monto del mismo varía según la importancia de la jugadora para el equipo. Así, las titulares reciben el monto más elevado, mientras que las jugadoras más jóvenes y recién llegadas perciben un estipendio menor. Cabe aclarar que en ninguno de los casos este estipendio es suficiente para sobrellevar los gastos diarios, por lo cual las jugadoras necesitan encontrar otros medios para subsistir. En algunos casos, sus familias las ayudan, pero en su mayoría las jugadoras deben llevar a cabo otras actividades para sostenerse. En este sentido, las experiencias de las jugadoras son muy diversas: algunas pasan muchas horas afuera de su casa ya que deben realizar largas jornadas laborales; a otras se les dificulta conseguir trabajos estables por el horario de entrenamiento; unas están imposibilitadas de asistir a la totalidad de los entrenamientos debido a sus trabajos; mientras que otras, a partir de su posición como jugadoras importantes del club, logran conseguir trabajo gracias a un dirigente de la institución. Esta variedad de casos da cuenta de la precariedad de la situación de las jugadoras, y la forma disímil en que la ausencia de un contrato las afecta a partir de su situación socio-económica y de su status en el club. De hecho, además del viático, Boca provee a un grupo de jugadoras, las más necesitadas, un bolsón de comida todas las semanas, y proporciona alojamiento a las jugadoras que provienen de otras provincias, en un departamento cercano al predio de entrenamiento.

Una estrategia adoptada por varias jugadoras consiste en "sumar viáticos", a partir de su participación en clubes de futsal donde reciben un viático que complementa el que reciben en Boca. Esta práctica es motivo de discusiones recurrentes ya que conlleva la posibilidad de generar inconvenientes para el desempeño de las jugadoras. Entre ellos, existe la posibilidad de que se superpongan los partidos de los distintos equipos en los que participa una jugadora. En una de tales ocasiones, una jugadora le explicaba a la delegada ${ }^{18}$ que si Boca jugaba el domingo a la

\footnotetext{
${ }^{18}$ Los/las delegados/as se ocupan de los temas administrativos y logísticos del equipo.
} 
FuLiA / UFMG - [...] las desigualdades estructurales en el fútbol de mujeres en Argentina

mañana se superponía con su partido de futsal en San Lorenzo. Dicha jugadora frente al cuestionamiento de una compañera que insistía en que debía elegir entre una situación u otra, contestó: "no, porque necesito los dos sueldos!".

Por otro lado, puede ocurrir que una jugadora se lesione jugando en otro club, debiendo llevar a cabo una rehabilitación que le impida participar de los entrenamientos y partidos en Boca. Presenciamos varias instancias en las cuales el cuerpo técnico y el cuerpo médico discutían al respecto, como en una ocasión en la cual una jugadora llegó al entrenamiento con un cuello ortopédico. La kinesióloga del club se lamentaba: "Nosotros vamos a tener serios, serios problemas. Entre el futsal y la Selección... la otra jugando al futsal, pumba, se golpeó la cabeza, no la tenemos por dos semanas". El delegado comparó la situación de Boca con la de UAI Urquiza, donde "si jugás en once, no jugás en otro lado. El técnico no las deja". Esta situación expone uno de los conflictos que plantea el hecho de que la práctica sea amateur. Se reconoce que la participación de las jugadoras en otros clubes es perjudicial para su rendimiento en Boca, pero se plantea la imposibilidad de exigirles exclusividad en tanto la institución no puede garantizarles un sostén económico.

En las entrevistas realizadas a jugadoras de distintos equipos, un tema recurrente es el del campo de juego y el horario dispuestos para sus entrenamientos. Casi todas las jugadoras plantearon que sus clubes priorizan a los varones -a los mayores, pero también a las divisiones juveniles-, y las obligan a entrenar en las peores canchas y horarios. Esta situación genera mayor indignación cuando las jugadoras pagan una cuota, como explicó Nicole respecto del club Sociedad Hebraica:

Teníamos la peor cancha de todas, y los chicos de primera jugaban en la mejor. Así tuvimos un montón de lesionadas, en la última parte del año se nos lesionaron tres. [...] Nosotras solicitamos que se hiciera rotación de cancha, y no nos hicieron rotación de cancha nunca. ¡Pagábamos la misma cuota! Entonces, ¿por qué a nosotras nos daban la peor cancha?

En Boca Juniors la situación es similar, ya que también se prioriza al fútbol masculino en lo que respecta a los campos de juego y los horarios de entrenamiento y partido. El club dispone de dos canchas de césped natural en el predio en que 
FuLiA / UFMG - [...] las desigualdades estructurales en el fútbol de mujeres en Argentina

entrenan las jugadoras, pero están reservadas para los equipos masculinos, por lo que el equipo de mujeres juega en una cancha de césped sintético. Además, los horarios de partidos y entrenamientos de las mujeres siempre dependen de los del masculino. Cuando el equipo masculino de Boca juega de local, el equipo femenino debe modificar su día de entrenamiento. En estos casos, el entrenamiento de las mujeres se superpone con el entrenamiento de las divisiones juveniles masculinas, por lo cual las mujeres deben practicar en un sector reducido de la cancha o mudarse a una de menores dimensiones. Incluso una jugadora nos comentó que en algunas ocasiones debieron entrenar en un estacionamiento del club porque la cancha estaba ocupada.

Otro punto que suelen destacar las jugadoras como problemático respecto de las condiciones en las que realizan su actividad es el de la indumentaria deportiva. Los dirigentes muchas veces les proporcionan camisetas con talles masculinos, inadecuadas para sus cuerpos, e incluso en algunas ocasiones les dan camisetas usadas previamente por los hombres. Al respecto, Nicole calificó este tipo de tratos como una forma de violencia y me expresó lo incómoda que se sentía con la ropa que les daba el club:

Nosotras teníamos las camisetas que habían pertenecido a los hombres, que nos quedaban grandes. No teníamos el talle femenino. [...] Qué se yo, usar una remera que me quedaba por el piso, o tener un short que nunca sabés qué talle pedirte, porque si te pedís el más grande te queda grande, y si te pedís el chiquito se te marca todo y... yo no jugaba muy cómoda con el equipo.

En cambio, en Boca, las jugadoras entrevistadas destacaron que usan la indumentaria adecuada. En este sentido, la delegada del fútbol femenino del club me explicó que en Boca "son muy estrictos" con el uso de la indumentaria actualizada de Boca, debido a los acuerdos con las marcas que lo sponsorean. Por este motivo todas las temporadas las jugadoras reciben las nuevas camisetas.

A partir de lo expuesto, queda claro que existen algunas condiciones estructurales que afectan a las jugadoras de fútbol de todos los equipos que participan en los torneos de AFA y que las ubican en inferioridad de condiciones respecto del fútbol masculino, que siempre es priorizado por sus clubes por considerarlo una 
FuLiA / UFMG - [...] las desigualdades estructurales en el fútbol de mujeres en Argentina

inversión redituable, mientras que la práctica de las mujeres es tomada como un gasto. Sin embargo, a partir de la comparación del caso de Boca Juniors con la experiencia de las jugadoras de otros equipos, se evidencia que existen diferencias entre los clubes y que las problemáticas que plantea la falta de profesionalización de la práctica son resueltas de distintas maneras entre las partes. Estas diferencias, a su vez, se expresan en los resultados obtenidos por los equipos en los torneos, donde los clubes que proporcionan las mejores condiciones a sus jugadoras obtienen los puestos más altos.

En última instancia estas diferencias son una consecuencia de la tensión entre el amateurismo y el profesionalismo que atraviesa el fútbol femenino en nuestro país. Esto es, si bien la práctica se plantea como amateur, los clubes llevan adelante estrategias para atraer y conservar a las mejores jugadoras. Esta acción se corresponde con lo que en nuestro país se denominó "amateurismo marrón", en tanto implica una motivación económica que no estaría presente en el amateurismo "puro". El ejemplo más claro de este "marronismo" se observa en el club UAI Urquiza, tal como lo explica Garton:

A través de la asignación de becas universitarias, viviendas, comidas, viáticos y trabajos part-time dentro de las empresas de Vaneduc, UAI Urquiza ha logrado atraer y mantener algunas de las jugadoras más habilidosas del país, de las cuales más de la mitad tiene experiencia en la selección, a nivel juvenil y/o mayor. ${ }^{19}$

Este profesionalismo encubierto también se observa cuando una jugadora desea cambiar de equipo. En estos casos, los clubes a veces actúan como si las jugadoras fueran de su propiedad y reclaman una compensación al club de destino, que no corresponde justamente por tratarse de una disciplina amateur. En este sentido, cabe mencionar el reciente episodio ocurrido a la futbolista Macarena Sánchez del club UAI Urquiza. En enero de 2018, Macarena fue despedida en medio de la temporada, dejándola sin posibilidad de jugar al fútbol durante 6 meses. Esto llevó a

\footnotetext{
${ }^{19}$ GARTON. Las Guerreras: Futbolistas, estudiantes, trabajadoras - un estudio sobre el fútbol de mujeres en el Club Deportivo UAl Urquiza.
} 
FuLiA / UFMG - [...] las desigualdades estructurales en el fútbol de mujeres en Argentina

que la jugadora denuncie el encubrimiento de la relación laboral que mantenía con el club bajo el rótulo de una práctica "amateur". A partir de la repercusión de este caso, Macarena Sánchez se convirtió en una referente en la lucha de las jugadoras por la profesionalización de su deporte.

\section{ConCLUSIón}

Desde abril de 2018 hasta la actualidad, el fútbol practicado por mujeres ha recibido una creciente atención de los medios de comunicación, al punto tal que incluso se ha revisado la invisibilidad histórica de las mujeres en el fútbol a partir del reconocimiento de las jugadoras pioneras. Por otra parte, la noticia relevante de 2019 ha sido el anuncio por parte de la AFA de la profesionalización de esta disciplina.

No obstante, pese a estos avances, el fútbol continúa exponiendo las dificultades que enfrentan las mujeres cuando se apropian de una práctica asociada culturalmente a los varones. El abanico de desigualdades se manifiesta de múltiples maneras. En este artículo, consideramos dos dimensiones para problematizar las dificultades de las mujeres a la hora de ser reconocidas como protagonistas legítimas del fútbol. Por un lado, analizamos las representaciones en un medio de comunicación deportivo de tirada nacional, que da cuenta de la posición subalterna que las futbolistas han tenido a lo largo de la historia del deporte, reforzando los estereotipos utilizados comúnmente en torno a las mujeres: ellas aparecen como madres, esposas e hijas; son sexualizadas; sus actuaciones deportivas son minimizadas. Por otro lado, describimos las condiciones en las que las jugadoras llevan a cabo su práctica de entrenamiento y competencias, sin contratos de trabajo debido al estatus amateur del fútbol femenino, pero con exigencias de un nivel profesional, que conviven con zonas grises de un intercambio de favores que no responden a un amateurismo puro.

Los resultados de nuestro trabajo resultan llamativos si pensamos que Argentina es un país netamente futbolero. No obstante, estos mismos datos nos llevan a pensar, siguiendo a Hargreaves, que el deporte, como cualquier otra instancia 
FuLiA / UFMG - [...] las desigualdades estructurales en el fútbol de mujeres en Argentina

cultural, encarna el conflicto; esto es, es un espacio de disputa por sus sentidos legítimos. ${ }^{20}$ ¿Quiénes han contado la historia del fútbol en Argentina hasta el momento? ¿Quiénes han tenido la palabra y la posición autorizadas para articular ese relato oficial? Los medios de comunicación masivos han jugado un rol importante en esta disputa al invisibilizar las prácticas deportivas de las mujeres, despojándolas de la posibilidad de protagonizar los relatos sobre la patria y los clubes -que son asociaciones deportivas, sociales y culturales, centrales en la vida cotidiana de nuestro país. En particular, el fútbol es un espacio de conflicto porque ha funcionado como reducto para la formación de masculinidades hegemónicas argentinas. Los estudios académicos, principalmente centrados en las investigaciones sobre varones, han reforzado la siguiente noción: "el fútbol es una práctica de varones” o, su contracara, "las mujeres no juegan al fútbol”.

Hoy, esta situación está en proceso de revertirse en la medida en que una serie de investigadores intentan remediar estos "olvidos", inscribiendo el análisis de la situación de las futbolistas argentinas en un contexto más amplio: el de las atletas en el deporte nacional y el de las mujeres en la sociedad. En este sentido, es necesario destacar que el acceso de las mujeres a actividades de ocio, recreativas y deportivas es muy limitado en comparación con el de los varones, y esta desigualdad se intensifica en el caso de las mujeres y niñas de sectores populares. En tanto no se modifiquen las condiciones estructurales que oprimen a las mujeres en los distintos aspectos de sus vidas (desigualdades referidas a las tareas de cuidado y limpieza del hogar, por ejemplo) no podrá lograrse una igualdad genuina en el deporte, más allá de los avances como la ya mencionada profesionalización del fútbol.

Finalmente, siguiendo a Beal, pensamos en un tipo de feminismo etnográfico que apunte a describir no sólo las condiciones de desigualdad, opresión y dominación de las mujeres, sino también las formas en que ellas negocian con estas condiciones estructurales, se adaptan a ellas o generan estrategias de resistencia. ${ }^{21} \mathrm{~A}$ su vez, reconocemos nuestra condición como la voz autorizada del conocimiento académico y

\footnotetext{
${ }^{20}$ HARGREAVES. Promesa y problemas en el ocio y los deportes femeninos.

${ }^{21}$ BEAL. Feminist Ethnographies in Sport and Leisure.
} 
la responsabilidad que conlleva esta posición en relación con la posibilidad de poner en un primer plano maneras múltiples y alternativas de expresar y vivir las identidades de género. ${ }^{22}$

\section{REFERENCIAS}

ALABARCES, Pablo. Fútbol y patria - el fútbol y las narrativas de la nación argentina. Buenos Aires: Prometeo, 2002.

ALVAREZ LITKE, Martín. Marcando la cancha: una aproximación al fútbol femenino desde las ciencias sociales. Cuestiones de sociología, n. 18, jun. 2018. ARAOZ ORTÍZ, Leandro; MOREIRA, Verónica. Prensa deportiva en Argentina. Construcciones identitarias y estilos discursivos del deporte en el diario Olé. Trama de la Comunicación, v. 20, n. 2, p. 111-124, jul.-dic. 2016.

ARCHETTI, Eduardo. Fútbol y ethos. Documentos e Informes de Investigación 7. Buenos Aires: FLACSO, 1984.

ARCHETTI, Eduardo. El potrero, la pista y el ring - las patrias del deporte argentino. Buenos Aires: Fondo de Cultura Económica, 2001.

BEAL, Becky. Feminist Ethnographies in Sport and Leisure. In: MANSFIELD, Louise; CLAUDWELL, Jayne; WHEATON, Belinda; WATSON, BecCy. (Orgs.). The Palgrave Handbook of Feminism and Sport, Leisure and Physical Education. London: Palgrave, 2018, p. 227-242.

BINELLO, Gabriela; CONDE, Mariana; MARTINEZ, Analía; RODRIGUEZ, María Graciela. Mujeres y fútbol: ¿territorio conquistado o a conquistar?, In: ALABARCES, Pablo. (Org.). Peligro de Gol - estudios sobre deporte y sociedad en América Latina. Buenos Aires: CLACSO, 2002, p. 33-55.

BINELLO; Gabriela; DOMINO, Mariano. Mujeres en el área chica. In: ALABARCES, Pablo; FRYDENBERG, Julio; DI GIANO, Roberto. (Orgs.). Deporte y sociedad. Buenos Aires: Eudeba, 1998, p. 211-226.

${ }^{22}$ BEAL. Feminist Ethnographies in Sport and Leisure. 
CONDE, Mariana. El poder de la razón: las mujeres en el fútbol. Nueva sociedad, v. 218,2008 , p. $122-130$.

CONDE, Mariana; RODRIGUEZ, María Graciela. Mujeres en el fútbol argentino: sobre prácticas y representaciones. Alteridades, v. 12, n. 23, p. 93-106, ene.-jun. 2002.

GARTON, Gabriela. Las guerreras: futbolistas, estudiantes, trabajadoras - un estudio sobre el fútbol de mujeres en el Club Deportivo UAI Urquiza. Tesis (maestría en Sociología de la Cultura y Análisis Cultural), IDAES/Universidad de San Martín, Buenos Aires, 2018.

GARTON, Gabriela; HIJOS, Nemesia. '“La deportista moderna': género, clase y consumo en el fútbol, running y hockey argentinos". Antípoda - Revista de Antropología y Arqueología, n. 30, p. 23-42, ene. 2018.

GOELLNER, Silvana; SILVA, Paula; BOTELHO-GOMES, Paula. A subrepresentação do futebol praticado por mulheres no jornalismo esportivo de Portugal: um estudo sobre a Algarve Women's Football Cup. Movimento, v. 19, n. 3, jul.-set. 2013.

IBARRA, Mariana. Lógicas del fútbol femenino: sin salario ni clubes. CONICET, Buenos Aires, 04 jun. 2018. http://bit.ly/2UY9tbN. Acceso en: 15 de mar. 2019.

KESSLER, Claudia Samuel. Futebol ou futebóis: é plural ou singular?. In: KESLLER, Claudia. (Org.). Mulheres na área: género, diversidade e inserções no futebol. Porto Alegre: UFGRS, 2016, p. 21-41.

HARGREAVES, Jennifer. Promesa y problemas en el ocio y los deportes femeninos. In: BROHM, Jean-Marie; BOURDIEU, Pierre; DUNNING, Eric; HARGREAVES, Jennifer; TODD, Terry; YOUNG, Kevin. (Orgs.). Materiales de sociología del deporte. Madrid: La piqueta, 1993, 109-132.

ROBLES, Magalí. Volver a la Historia. EI Equipo Deportea online, Buenos Aires, 08 mar. 2018. Disponible en: http://bit.ly/2UYoZUI. Acceso en: sep. 2018.

Recebido para publicação em: 10 maio 2019. Aprovado em: 26 maio 2019. 